\section{Treatment of gingival lichen with free palatal grafts}

Axéll T, Henriksen BM J Oral Pathol Med 2007; 36: 105-109

Most grafts were fully successful in treating recalcitrant lesions.

Lichen planus may present on the buccal gingiva as a desquamative gingivitis and can be resistant to treatment with medications such as steroids. Following one report of successful treatment with grafting, this study reports results from 12 Norwegian patients with recalcitrant lesions (unspecified number confirmed by biopsy) who received 20 healthy grafts from the hard palate, trimmed to replace buccal gingiva.

Grafts were observed for 5-97 months (mean 32). On a 3 point scale of healing, 12 patients were healed completely, 6 healed but with some erythema and no symptoms, and 2 improved but had extensive erythema and/or symptoms. The authors discuss diagnostic validity and state that biopsy confirmation of the condition may be complicated by a concurrent plaqueinduced gingivitis.

\section{DOI: $10.1038 /$ bdj.2007.536}

\section{ORTHODONTICS}

\section{Patients' expectations of orthodontic treatment: part 2 - findings from a questionnaire survey}

Sayers MS, Newton JT J Orthod 2007; 34: 25-35

Patients and parents had similar expectations, but parents were more realistic in estimating length of treatment.

There is little good information on what young patients expect before receiving orthodontic treatment. In this study, 50 patients (age 12-14), and one parent of each, completed a previously validated questionnaire prior to orthodontic consultation.

Similar proportions of parents and children agreed on most questions. Parents had significantly lower expectation of an appliance at the first visit, and children expected greater restrictions on what they could eat or drink. Children also expected a quicker completion of treatment.

There were also significantly different responses from White and non-White children. White children considered an appliance at the first visit to be very unlikely, headgear to be less likely, and the appliance unlikely to be painful. Non-White children also were more expectant of a short duration to treatment, a positive attitude to their appliance from others, and improved speech ability following treatment. Parents too had some differences according to ethnic group.

\section{ORAL SURGERY; PHYSIOLOGY}

\section{Magnetic resonance imaging assessment of airway status after orthognathic surgery}

\section{Meisami T, Musa M et al. Oral Surg 2007; 103: 458-463}

There was no significant airway compromise in a variety of different procedures.

Airway obstruction following orthognathic surgery (OGS) is a rare, life-threatening complication about which little has been reported. In this study, the airways of 40 patients undergoing OGS were assessed pre-operatively and 24-48 hours subsequently (expected time of maximum oedema), using magnetic resonance imaging (MRI). Scans were examined in masked (blinded) conditions.

The OGS procedures, alone or in combination, were Le Fort I osteotomy (34 patients), bilateral sagittal split osteotomy (23) and genioplasty (25). In no case was airway oedema or compromise identified by MRI, despite clinically obvious oedema of lips, cheeks and upper necks. The authors comment that in the absence of other clinical signs, routine admission to intensive care and overnight intubation appear unnecessary, and that intermaxillary fixation is not needed where rigid fixation has been used.

DOI: $10.1038 / b d j .2007 .537$

ORTHODONTICS

\section{Long-term follow-up of orthodontically treated deep bite patients}

Schütz-Fransson U, Bjerklin K et al. Eur J Orthod 2006; 28: 503-512

Stability was good 11 yrs after treatment.

Overbite correction may relapse, and this may be correlated with factors such as protrusion of the mandibular anterior segment and subsequent development of the jaws. In this study, 30 patients treated for an overbite of $4.5 \mathrm{~mm}$ or more were compared with 32 normal control subjects.

Most patients had Class II malocclusion, 23 were treated with edgewise appliances and 7 with Andresen activators. No extractions were made, and 7 patients received no retention. Subjects were assessed at mean ages 12 yrs (pre-treatment), 15 (post), 19 and 26, and controls at 9, 14, 21 and 31 .

Treatment reduced overbite from a mean 5.8 to $2.8 \mathrm{~mm}$. Mean bite relapse in patients was $0.8 \mathrm{~mm}$ at the 4 th assessment, but overbite was still within the normal range, while overbite in controls reduced by $0.7 \mathrm{~mm}$. Mandibular incisor space also reduced slightly. There were modest differences between groups, but the authors concluded that treatment results were stable.

DOI: 10.1038/bdj.2007.539 